\title{
Comparative study of red blood cell morphology in peripheral smear and automated cell counter
}

\author{
Goyal S.C. ${ }^{1}$, Shah N.L. ${ }^{2}$, Shah F.R ${ }^{3}$, Shah J. $M^{4}$. \\ ${ }^{1}$ Dr. Sunita C. Goyal, ${ }^{2}$ Dr. Nilay L. Shah, ${ }^{3}$ Dr. F.R. Shah, ${ }^{4}$ Dr. J. M. Shah, ${ }^{1,2}$ Assistant Professor, Department of \\ Pathology, GMERS Medical College, Himmatnagar. ${ }^{3}$ Associate Professor, ${ }^{4}$ Professor \& H.O.D.AMC MET Medical \\ College, Maninagar, Ahmedabad, Gujarat 380008.
}

Corresponding Author: Dr. Nilay L. Shah, Pathology Department, GMERS Medical College, Himmatnagar, Sabarkantha, Gujarat, India. E-mail id-dr.nilay2020@gmail.com

\begin{abstract}
Background: Automated cell counters are a very important part of pathology laboratory for evaluation of complete blood count $(\mathrm{CBC})$. They also provide RBC histograms to interpret different morphological variations of Red Blood Cells. These histograms and other parameters have been found very useful in diagnosing various hematological conditions and Red Blood Cell disorders if they are correctly interpreted. Examination of peripheral smears is still the gold standard for diagnosing some of the RBC disorders which might not be diagnosed otherwise by automated cell counters. They play an important role in quality check of automated analyzers. Materials and Methods: In this prospective study we have taken 200 samples over a period of 6 months. We did comparative study between RBC histograms obtained by automated hematology analyzer and peripheral blood smears stained by field stain. We have discussed morphological variations of red blood cells and their characteristic changes in respective RBC histograms. Result: Out of 200 samples of RBC histogram interpretation, 138 cases showed correlation with peripheral smear findings while 62 cases showed discrepancies. Conclusion: Microscopic examination of peripheral blood smear still remains gold standard for diagnosis of various hematological conditions.
\end{abstract}

Keywords: RBC morphology, Peripheral blood smear, Red cell disorders, Automated cell counter

\section{Introduction}

Peripheral blood smear examination has been an important part of investigation for various hematological disorders since decades and also major diagnostic tool especially for etiopathological work up of different hematological disorder. The automated hematology analyzer has replaced the traditional manual methods for measuring various hematological parameters as the initial screening method in most of the hospital nowadays [1]

Along the years there have been different studies from time to time for assessing the utility and accuracy of automated cell counter generated parameters in general as well as with respect to diagnose specific types of anemia. This study is an attempt to standardize few automated red cell parameters and to compare these with microscopic examination of peripheral blood smear. Automated cell counter provide histogram of RBCs which give us important clue regarding particle

Manuscript received: $5^{\text {th }}$ February 2019

Reviewed: $14^{\text {th }}$ February 2019

Author Corrected: $18^{\text {th }}$ February 2019

Accepted for Publication: $22^{\text {nd }}$ February 2019 size, volume. This RBCs histogram if interpreted along with other important RBCs indices like Red cell distribution width (RDW) and mean corpuscular volume (MCV), have been found very useful in work up of many hematological disorders and may provide major diagnostic clue in condition like anemia, thalassemia [2-5]. In addition, RBCs histogram most widely used with peripheral blood smear to monitor and interpret abnormal morphological variation of red blood cells like, dimorphic red cells.

\section{Aims and Objectives}

Aims and objective of our study is to compare accuracy of RBC histograms shown by our 5 part cell counter to that of microscopy examination of blood film stain by field stain.

\section{Materials and Methods}

This is a prospective study over a period of 6 months. In this comparative study of RBC morphology, we have collected total of 200 samples which have been received 


\section{Original Research Article}

in GMERS Medical College, Himmatnagar and are evaluated by both, histograms obtained through Horiba 5 part cell analyzer (automated hematology analyzer) and peripheral blood smear which is stained by Field stain. Patients with normal hemoglobin are excluded in this study.

Study site- The study was conducted at the central laboratory, Pathology Department, GMERS Medical College, Himmatnagar. Duration of study was 6 months from January 2018 to June 2018.

Study design- Observational study.
Case selection- The study was carried out on all anemic samples as per WHO reference range.

Inclusion criteria: All anemic samples as per WHO reference range.

Exclusion Criteria: Children below 10 years were excluded.

Sample size- Sample size of present study is 200 .

Ethical permission- Permission was taken from Institutional Ethics Committee to conduct this study.

Statistical Analysis: The data was analysed using the Microsoft Excel 2007.

\section{Results}

We have studied 200 samples. Among them 138 cases show correlation between histogram to that of the peripheral blood smear examination. Distribution of such cases is shown in table 1.

Table-1: Distribution of cases which show correlation.

\begin{tabular}{|l|c|c|}
\hline Cases & Total No & \% \\
\hline Iron Deficiency Anemia & 40 & 28.9 \\
\hline Megaloblastic anemia & 20 & 14.4 \\
\hline Alcoholic liver disease & 10 & 7.2 \\
\hline High reticulocytes count & 18 & 13.1 \\
\hline Post Iron deficiency anemia therapy & 22 & 15.9 \\
\hline Beta Thalassemia major & 12 & 8.6 \\
\hline Beta Thalassemia minor & 16 & 11.5 \\
\hline Total & $\mathbf{1 3 8}$ & $\mathbf{1 0 0}$ \\
\hline
\end{tabular}

62 cases out of 200 samples did not show correlation between histogram to that of peripheral blood smear examination. Distribution of such cases is shown in table 2.

Table-2: Distribution of case which did not show correlation.

\begin{tabular}{|l|c|c|}
\hline Cases & Total No & \% \\
\hline Platelets clumps & $\mathbf{2 3}$ & $\mathbf{3 7 . 1}$ \\
\hline Giant platelets & $\mathbf{1 0}$ & $\mathbf{1 6 . 1}$ \\
\hline Cold agglutinations & $\mathbf{6}$ & $\mathbf{9 . 6}$ \\
\hline High leucocytes count & $\mathbf{5}$ & $\mathbf{8 . 1}$ \\
\hline High Nucleated RBCs & $\mathbf{8}$ & $\mathbf{1 2 . 9}$ \\
\hline Post transfusion cases & $\mathbf{8}$ & $\mathbf{1 2 . 9}$ \\
\hline Chronic lymphocytic leukemia & $\mathbf{2}$ & $\mathbf{3 . 2}$ \\
\hline Total & $\mathbf{6 2}$ & $\mathbf{1 0 0}$ \\
\hline
\end{tabular}

Interpretation of histograms, in conjunction with the numerical data can be clinically useful in the diagnosis and followup of many hematological and non-hematological conditions. We have to look after shape, centre, and spread of histogram along with overall pattern for correct reading of it. This pattern should be read along with other reference normal curve and/or to be confirmed microscopically by expert. Symmetric and skewed shapes are observed in RBC histogram which are seen and easily identified but others variation in shape may be more challenging, especially when two populations of red cells are present and such cases should be confirmed by microscopy. Direct inspection of the distribution curve gives us information about size of red blood cells and their variation microcytes and macrocytes, however the estimation of the number of cells from the histogram should be avoided as erroneous results can arise because the frequency curve shows only the relative information and not give us the actual number of cells in each size range. [6] 


\section{Original Research Article}

Iron deficiency anaemia (Fig 1a) and beta thalassemia trait (Fig 1b) are the leading causes for microcytic anemia in our country, and they are easily identified and classified on the basis of RBC histogram. The red cell histogram is shifted toward left, and the percentage of microcytes is increased in both disorders. Although their histograms are similar, variation of size of red blood cells which as measured by the RDW (red cell distribution width) can easily differentiates two of them. Iron deficiency anaemia have characteristic feature of elevated RDW where as in thalassemia trait has RDW within normal range. We have studied 40 cases of iron deficiency anemia and 16 cases of thalassemia trait and easily differentiated them. We found a significant increase in mean RDW among iron deficiency anemia (18.37\%) compared to beta thalassemia trait group $(16.55 \%)$

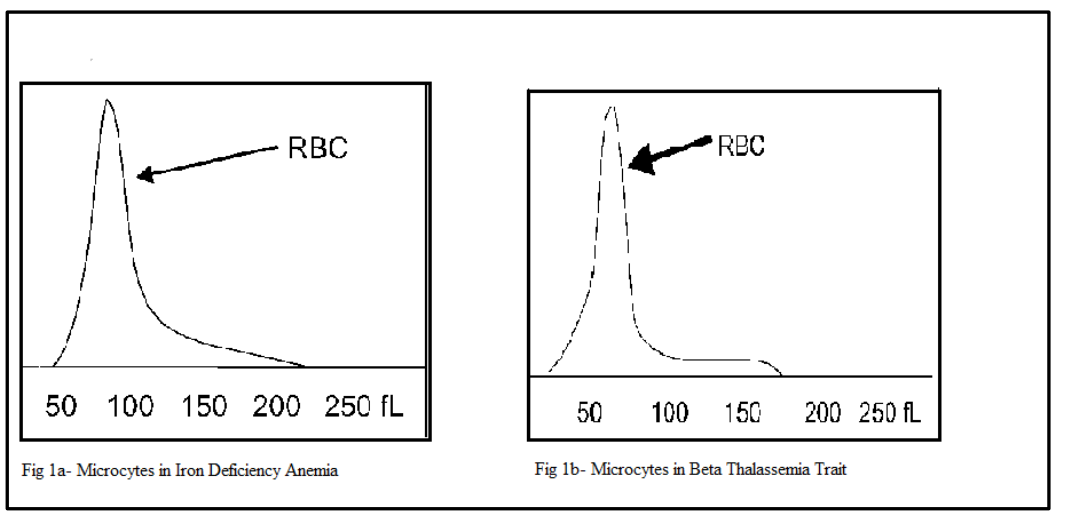

Figure 1

We found that Red blood cells having increase in size which are called as macrocytes are found in number of conditions like megaloblastic anemia, alcoholic liver disease, drug induced and in cases of hypothyroidism.

Increase in red blood cell is called as macrocytes. Megaloblastic anemia and alcoholic liver disease are main etiology for it. We studied that in megaloblastic anemia, there is small peak on the left of the macrocytic peak because of fragmented RBCs and very small cells and having high variation in size of red blood cells indicated by high RDW (Fig 2a ) whereas alcoholic liver disease show only single macrocytic peak in graph (Fig $2 b$ ). We have found 20 cases of megaloblastic anemia which have Widespread in the macrocytic zone and 10 cases of alcoholic liver disease which have closely clustered in macrocytic zone in our study.

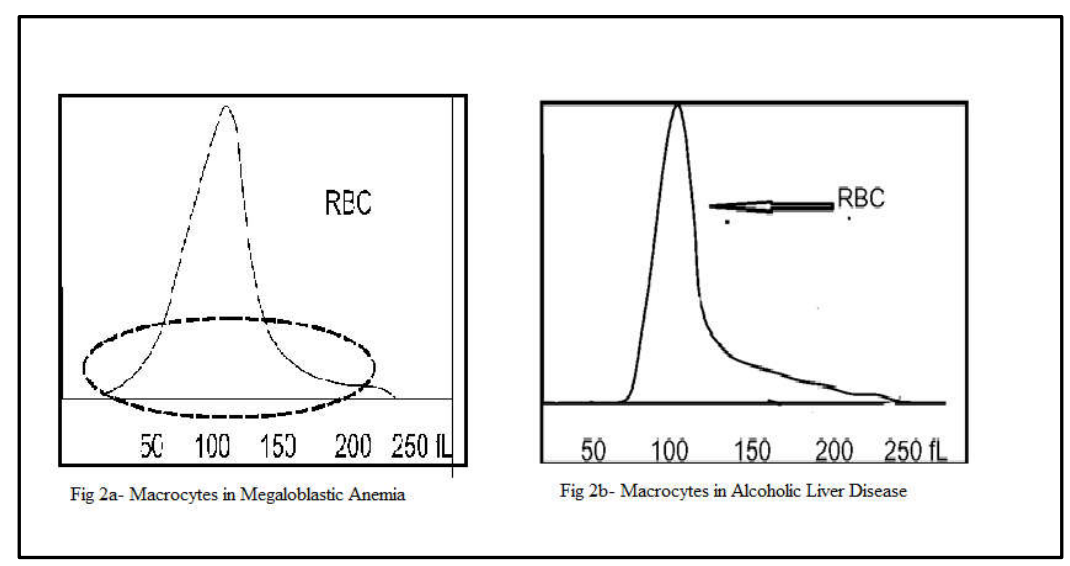

Figure 2

In Beta-thalassemia major, (Fig 3a) There is presence of skewed curve on histogram. This is because of high number of nucleated RBCs and extremely heterogeneous morphology which are associated with high RDW. There are increase number of microcytes is seen at the beginning of the histogram. This is because of presence of red cell fragments, nucleated RBCs and microcytic red cells. We found 12 cases of thalassemia major.

In reticulocytosis (Fig 3b) the histogram is bimodal and is skewed to the right. We found 18 cases of high reticulocytes count. 


\section{Original Research Article}

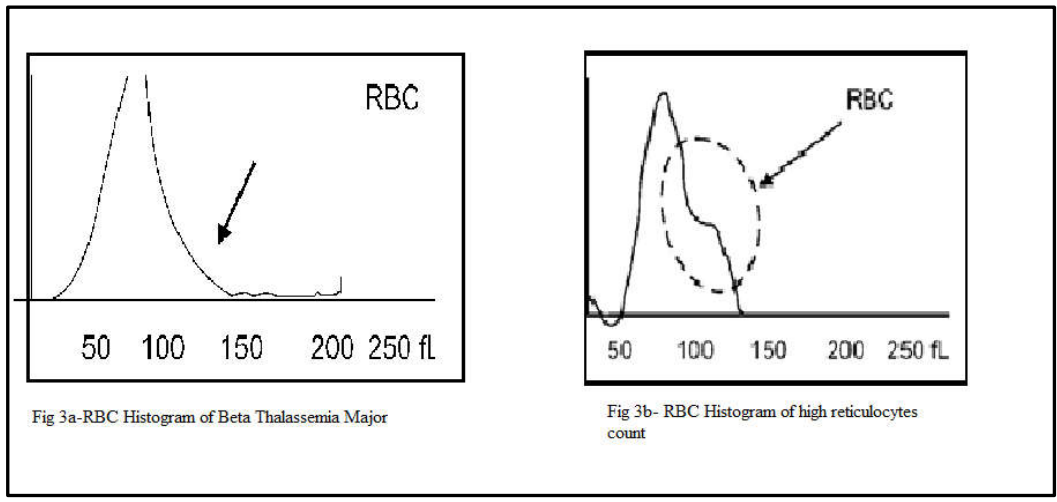

Figure 3

In samples of Patients who are on therapy of iron deficiency anemia( Fig 4) shows second population of normocytic cells in RBC histogram along with microcytic peak because of Iron deficiency. We found 22 cases of such in our study.

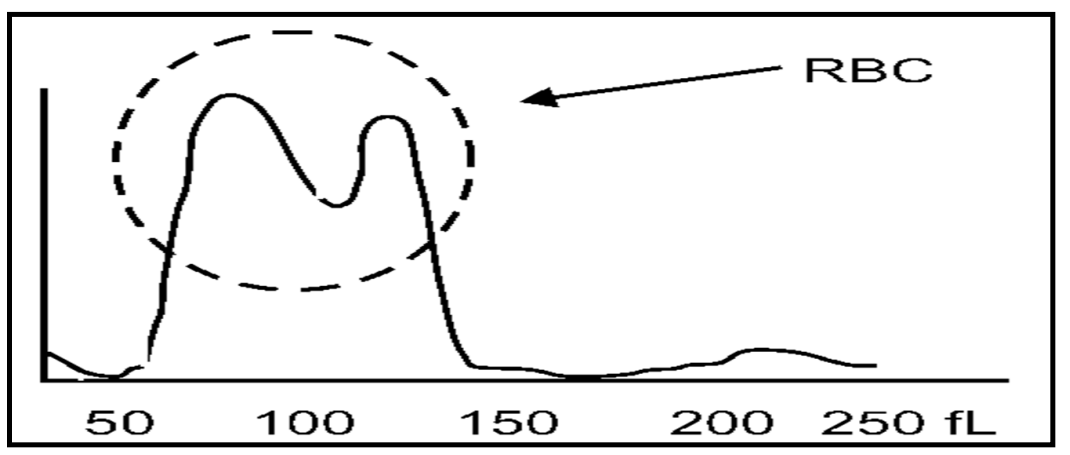

Figure 4

In case of cold agglutination, bimodal RBC histogram peaks at wide apart distance of $90 \mathrm{fL}$ and $150 \mathrm{fL}$ is path gnomonic of red cells agglutinin. Other parameters should be look after like very high MCV, MCHC and markedly increased RDW in such cases. And for definitive diagnosis we should have to do microscopy of peripheral smear. We found 6 cases of cold agglutinin which are confirmed by microscopy.

Cases of platelets clumps and giant platelets are misinterpreted and plotted in histogram as micro erythrocytes so that correction was done by smear review. We found 23 cases of platelets clumps and 10 cases of giant platelets which are confirmed by microscopy.

Cases of high white blood cell count or post blood transfusion therapy are having misleading results on RBC histogram because they show multiple peaks. Such cases should be confirmed by microscopy. We found 7 cases of such in our study.

\section{Discussion}

The Coulter principle and Coulter counter was real discovery in hematology field, and the prolific Coulter's revolutionized laboratory procedures are very important in etiopathological work up for different hematological condition. Coulter Principle stated that Sizing and counting cells by detecting and measuring changes in electrical resistance when a cell passes through a small aperture.

A histogram is a vertical bar chart which gives us so much information. The volume histogram reflects the size of any cell which is found in that size range. When the volume sizes between $25 \mathrm{fL}$ and 250 fLoccurs, that cell is counted as erythrocytes by instrument. RBC histogram is a symmetrical bell-shaped curve. The area of the peak is used to calculate the different $\mathrm{RBC}$ parameters like red cell distribution width (RDW) and mean corpuscular volume (MCV). Norma area for RBCs represents $60 \mathrm{fL}$ to $125 \mathrm{fL}$.

If the RBCs become enlarge in size as in case of macrocytic anemia, the curve in RBC histogram will shift toward the right and if the RBCs become small in size as in case of microcytic anemia, the curve in RBC 
Original Research Article

histogram will shift to the left. There are certain variation in graph occur like if the histogram curve is bimodal (Camel humps) having two or more peak then there are two populations of red blood cells which occur when a patient received a blood transfusion, patients on treatment particularly in case of iron deficiency anemia, cold agglutinin disease, anemia with different size cell populations. Interpretation of the RBC histogram, along with other hematological parameters like RBC count, $\mathrm{HB}, \mathrm{HCT}, \mathrm{MCH}, \mathrm{MCHC}$ and RDW can be very handy in diagnosis of various $\mathrm{RBC}$ disorders.

The RBC histogram in our study are compared with that of $\mathrm{RBC}$ histogram which are given in book of "The $\mathrm{ABC}$ of CBC by DP Lokwani [7]. In certain conditions like post-transfusion therapy or infection or tumor anaemia or extreme leukocytosis which are showing multiple peaks in RBCs histogram and they are very difficult to interpret. We can easily differentiate thalassemia trait to that of iron deficiency anemia as formers have low MCV along with normal RDW while in case of iron deficiency anemia there is high RDW. In case of cold agglutination, the frequency curves may vary in shapes. It disappears when cold agglutinin samples are incubated at room temperature or incubated at 37-degree temperature. Histograms obtained in this study shows similar result.

We found that patients with macrocytic anaemia due to megaloblastic anemia showing small peak on the left of the macrocytic peak because of fragmented RBCs and very small cells and having high RDW where as alcoholic liver disease show only single macrocytic peak in graph, which are similar to histogram present in book which mentioned earlier.

In $\mathrm{RBC}$ histograms, size ranges are between $24 \mathrm{fL}$ and $360 \mathrm{fL}$. Cell counter analyzer counts every cell with size between $36 \mathrm{fL}$ and $360 \mathrm{fL}$ as red cells. Those cells which range between $24 \mathrm{fL}$ to $36 \mathrm{fL}$ are rejected as red blood cell by analyzer. Normally, the histogram below $36 \mathrm{fL}$ are generally clear, but in certain conditions like fragmented red cells, non lysed RBCs, giant platelets, platelet clumps, bacteria, parasitic infection, and other interfering substances such as cryoglobulinemia, cold agglutinin disease, the histogram may interfere normal distribution curve.

Number of factors like red cell agglutination, alteration in red cell shape, and inclusion of leukocytes, inclusion in red blood cells like parasite infection, $\mathrm{Hb} \mathrm{H}$ inclusion may affect the histogram. These factors, in one way or another, influence the histogram's appearances and accordingly will have a erroneous effect on histogram. [8] To reduce the effect of these problems, every manufacturers design their instruments and reagent systems to specifically prevent and correct for interferences which mentioned above. They develop mathematical algorithms for particle counting and produce numeric data, graphic data, scatter plots, and interpretative comments that will assist or alert the users to potential incorrect results $[9,10]$.

From this study we concluded that RBC histograms assist us in prediction of smear picture \&correct interpretation of histogram gives better idea about etiopathological workup of various hematological conditions and save precious time of pathologist. From reading histograms, we can have better idea about what to expect when we actually evaluate the peripheral blood film by microscopy. The speed, accuracy and reliability of the modern analyzers allow us to analyze very large number of complete blood count analysis within very short period of time which is impossible in case of manual reporting. It gives us enough time to evaluate abnormal blood films, consider diagnostic clues and correlate clinical findings to histograms. They facilitate us to report all samples with confidence and efficiency and all of which increase the standard of patient health care.

\section{Conclusion}

RBC Histogram is an important tool of diagnosis when correct interpretation of curve is combined with findings of blood count parameters like red cell distribution width and red cell indices. By observing these curves we could give presumptive diagnosis of presence of fragments in blood, microcytic, macrocytic or dimorphic red cells. Histograms along with Blood indices and hemoglobin value will guide us about RBC morphology. Histograms are useful tool for technologists as it could guide them that which cases need actual detailed peripheral smear examination by experts.

We concluded that though automated analyzers reduce overall workload by its advances of graphical representation, it should be confirmed by microscopy. Microscopy of peripheral blood film still remains gold standard for diagnosis of various hematological conditions.

\section{Contribution from the author}

- Dr. Sunita Goyal: Data collection, analysis and preparation of manuscript.

- Dr. Nilay Shah: Analysis and preparation of manuscript \& critical revision.

Findings: Nil; Conflict of Interest: None initiated

Permission from IRB: Yes 


\section{Reference}

1.Lantis KL, Harris RJ, Davis G, et al. Elimination of instrument-driven reflex manual differential leukocyte counts. Optimization of manual blood smear review criteria in a high-volume automated hematology laboratory. Am J Clin Pathol. 2003 May;119 (5):65662. DOI: 10. 1309/VH1K-MV8W-B7GB-7R14

2. Bessman JD, Gilmer PR Jr, Gardner FH. Improved classification of anemias by MCV and RDW. Am J Clin Pathol. 1983 Sep;80(3):322-6.

3. Williams LJ. Cell histograms: New trends in data interpretation and cell classification. Journal of medical technology. 1984;1(3):189-97.

4. Fossat C, David M, Harle JR, Sainty D, Horschowski $\mathrm{N}$, Verdot JJ, Mongin M. New parameters in erythrocyte counting. Value of histograms. Archives of pathology \& laboratory medicine. 1987 Dec;111 (12): $1150-4$.

5. Lawrence A, Young M, Cooper A, Turner E. Red cell histograms in the diagnosis of diseases. Hematology
Beyond the Microscope. New York, NY: Technicon Instruments. 1984:155-64.

6. Beckman Coulter LH. 780 on line IB072841. Beckman Coulter Education Center, Miami Lakes, FL. 2007.

7. Lokwani DP. The $\mathrm{ABC}$ of $\mathrm{CBC}$ : Interpretation of complete blood count and histograms. JP Medical Ltd; 2013 May 30.

8. Rowan RM. Blood Cell Volume Analysis: A New Screening Technology for the Haematologist. Albert Clark; 1983.

9. Steele BW, Wu NC, Whitcomb CL. White blood cell and platelet counting performance by hematology analyzers:a critical evaluation. Laboratory Hematology. 2001 Jan 1;7:255-66.

10. ART BT. High mean corpuscular hemoglobin concentration: Its causes and effects on automated CBC results. Canadian Journal of Medical Laboratory Science. 2007 May 1;69(3):113.

\section{How to cite this article?}

Goyal S.C, Shah N.L, Shah F.R, Shah J. M. Comparative study of red blood cell morphology in peripheral smear and automated cell counter. Trop J Path Micro 2019;5(2):88-93.doi:10.17511/jopm.2019.i02.07. 